Dark energy, non-minimal couplings and the origin of cosmic magnetic fields

This article has been downloaded from IOPscience. Please scroll down to see the full text article.

JCAP12(2010)025

(http://iopscience.iop.org/1475-7516/2010/12/025)

View the table of contents for this issue, or go to the journal homepage for more

Download details:

IP Address: 147.96.14.16

The article was downloaded on 27/06/2013 at 19:23

Please note that terms and conditions apply. 


\title{
Dark energy, non-minimal couplings and the origin of cosmic magnetic fields
}

\author{
Jose Beltrán Jiménez and Antonio L. Maroto \\ Departamento de Física Teórica, Universidad Complutense de Madrid, \\ 28040, Madrid, Spain \\ E-mail: jobeltra@fis.ucm.es, maroto@fis.ucm.es
}

Received November 4, 2010

Accepted December 14, 2010

Published December 27, 2010

\begin{abstract}
In this work we consider the most general electromagnetic theory in curved spacetime leading to linear second order differential equations, including non-minimal couplings to the space-time curvature. We assume the presence of a temporal electromagnetic background whose energy density plays the role of dark energy, as has been recently suggested. Imposing the consistency of the theory in the weak-field limit, we show that it reduces to standard electromagnetism in the presence of an effective electromagnetic current which is generated by the momentum density of the matter/energy distribution, even for neutral sources. This implies that in the presence of dark energy, the motion of large-scale structures generates magnetic fields. Estimates of the present amplitude of the generated seed fields for typical spiral galaxies could reach $10^{-9} \mathrm{G}$ without any amplification. In the case of compact rotating objects, the theory predicts their magnetic moments to be related to their angular momenta in the way suggested by the so called Schuster-Blackett conjecture.
\end{abstract}

Keywords: magnetic fields, dark energy theory 


\section{Contents}

1 Introduction 1

2 Generalized electromagnetism $\quad 2$

3 Consistency and stability 2

4 Cosmological evolution 4

5 Effective electromagnetic current: gravitational magnetism 5

$\begin{array}{llr}6 & \text { Discussion } & 6\end{array}$

\section{Introduction}

The origin of the magnetic fields observed in galaxies and galaxy clusters with large coherence lengths and strengths around $10^{-6} \mathrm{G}$ still remains an open problem in astrophysics [1] (recent works [2] also show evidence for the existence of extragalactic magnetic fields with strengths above $3 \times 10^{-16} \mathrm{G}$ ). Two different types of mechanisms have been proposed for the generation of such fields. On one hand, we have the primordial field hypothesis, i.e. relic fields from the early universe with comoving strengths around $10^{-10}-10^{-12} \mathrm{G}$ are amplified to the present values in the protogalactic collapse. On the other, much weaker fields around $10^{-19} \mathrm{G}$ at decoupling time could have been amplified by the galactic rotation through a dynamo mechanism. In both cases, preexisting seed fields are required. In fact, there are also several proposals for the generation of fields which could seed a galactic dynamo. They include astrophysical mechanisms [3], production during inflation [4], in phase transitions [5], by spontaneous breaking of Lorentz invariance [6] or by metric perturbations [7]. Nevertheless, it has been argued that the timescales for dynamo amplification may be too long to explain the observed fields in young objects [1]. In addition, the origin of the stronger large-scale seeds in the primordial approach is even more problematic.

A very interesting framework for magnetic field generation is the possibility that the standard electromagnetic theory could be modified in the presence of gravity. Thus in [4], couplings of the electromagnetic field to the space-time curvature were proposed as a way of producing magnetic fields during inflation. In this paper we will consider a generalized electromagnetic action in curved space-time, including also non-minimal couplings. The crucial difference with respect to previous works is that we allow for the presence of a homogeneous temporal electromagnetic background potential. This is motivated by the fact that, as has been recently shown [8], the presence of temporal electromagnetic potentials on cosmological scales could play the role of dark energy. Indeed, this type of fields can be amplified from quantum fluctuations during inflation in a completely analogous way to metric perturbations. The initial amplitude of the field being given by $\left\langle A_{0}^{2}\right\rangle^{1 / 2} \sim H_{I}$, where $H_{I}$ is the value of the Hubble parameter during inflation. The field is then shown to grow linearly in time in the matter and radiation eras, the corresponding energy density on cosmological scales behaving as a cosmological constant. Interestingly, the predicted value of the cosmological constant agrees with observations provided inflation took place at the electroweak scale. In such a 
case, the present value of the background field would be $\bar{A}_{0} \simeq 0.3 M_{P}$. Here we show that the non-minimal coupling of the temporal background to the space-time curvature implies that the energy-momentum density of any matter/energy distribution generates an effective electromagnetic current, even for neutral sources. This allows to establish a natural link between dark energy and the origin of cosmic magnetic fields.

\section{Generalized electromagnetism}

Let us consider the most general expression for the electromagnetic action in the presence of gravity, including all the possible terms leading to linear second order differential equations:

$$
S=\int d^{4} x \sqrt{-g}\left[-\frac{1}{4} F_{\mu \nu} F^{\mu \nu}+\frac{\lambda}{2}\left(\nabla_{\mu} A^{\mu}\right)^{2}+\sigma R_{\mu \nu} A^{\mu} A^{\nu}+\omega R A_{\mu} A^{\mu}\right] .
$$

Notice that this expression does not contain any dimensional parameter or potential term. The minimal case with $\sigma=\omega=0$ was studied in detail in [8] and the possibility of generating cosmic magnetic fields in this case has been considered recently in [9]. In this action, the $\lambda$ parameter can be fixed by choosing a normalization of the non-transverse modes and $\sigma$ and $\omega$ are arbitrary dimensionless constants. In order to fix them, we will consider the weak-field limit of the theory. Thus, the space-time metric can be written as a small perturbation around Minkowski space-time, $g_{\mu \nu}=\eta_{\mu \nu}+h_{\mu \nu}$ and the electromagnetic potential reads $A_{\mu}=\bar{A}_{\mu}+a_{\mu}$ with $\bar{A}_{\mu}=\bar{A}_{0} \delta_{\mu}^{0}$ and $\bar{A}_{0}$ constant. The background electromagnetic field is determined by the corresponding cosmological value and therefore it could evolve on cosmological timescales. However, for local experiments it is a good approximation to assume it constant (in agreement with the flat space-time background). Notice that the electric and magnetic fields associated to $\bar{A}_{\mu}$ identically vanish. The corresponding Maxwell equations obtained from (2.1) read to first order:

$$
\partial_{\nu} F^{\mu \nu}+\lambda \partial^{\mu}\left(\nabla_{\nu} A^{\nu}\right)_{(1)}=J_{g}^{\mu} .
$$

where $F_{\mu \nu}=\partial_{\mu} a_{\nu}-\partial_{\nu} a_{\mu},\left(\nabla_{\nu} A^{\nu}\right)_{(1)}$ denotes the contribution to first order and the nonminimal terms give rise to an effective current given also to first order by: $J_{g}^{\mu}=2\left(\sigma R_{(1)}^{\mu \nu}+\right.$ $\left.\omega R_{(1)} \eta^{\mu \nu}\right) \bar{A}_{\nu}$. Imposing this effective current to be conserved, i.e. $\partial_{\mu} J_{g}^{\mu}=0$, we obtain $\sigma=-2 \omega$, i.e. the non-minimal coupling must involve the conserved Einstein tensor $G_{\mu \nu}=$ $R_{\mu \nu}-\frac{1}{2} R g_{\mu \nu}$. Notice also that conservation implies that taking the divergence of (2.2) we get $\square\left(\nabla_{\mu} A^{\mu}\right)_{(1)}=0$, i.e. to first order it is possible to impose the Lorenz condition $\nabla_{\mu} A^{\mu}=0$ at the classical level as in ordinary electromagnetism, so that the $\lambda$ term disappears. Thus, for weak gravitational fields we recover ordinary Maxwell electromagnetism, the only difference is the appearance of a gravitationally-generated electromagnetic current. Notice that this current is only present provided the background electromagnetic potential is non-vanishing and in the presence of space-time curvature.

According to the previous discussion, the form of the action will be given by:

$$
S=\int d^{4} x \sqrt{-g}\left[-\frac{1}{4} F_{\mu \nu} F^{\mu \nu}+\frac{\lambda}{2}\left(\nabla_{\mu} A^{\mu}\right)^{2}+\sigma G_{\mu \nu} A^{\mu} A^{\nu}\right]
$$

\section{Consistency and stability}

This theory is a particular case of the more general class of vector-tensor theories [10]. These theories usually give rise to modifications of the gravitational interaction at small (Solar 
System) scales which are encoded in the parameters of the corresponding Parametrized PostNewtonian (PPN) approximation [10, 11]. For this particular case, the PPN parameters are: $\gamma-1 \simeq-16 \pi G \sigma A_{\odot}^{2}, \beta-1 \simeq-16 \pi G \sigma A_{\odot}^{2}, \alpha_{1} \simeq-64 \pi G \sigma A_{\odot}^{2}, \alpha_{2} \simeq-16 \pi G \sigma A_{\odot}^{2}$, where $A_{\odot}$ is the background amplitude at Solar System scales and we have assumed $|\sigma| \ll 1$, keeping only the leading order in the expansion. The most stringent constraint on the PPN parameters is $|\gamma-1| \leq 2.3 \times 10^{-5}$, which imposes the corresponding limit on $\left|\sigma A_{\odot}^{2}\right|$. If we assume that the amplitude of the electromagnetic field at Solar System scales resembles the cosmological value $A_{\odot} \simeq \bar{A}_{0} \simeq 0.3 M_{P}$, we obtain the constraint $|\sigma| \lesssim 10^{-5}$.

Let us now study the stability of the theory by analyzing the behavior of the inhomogeneous perturbations around the Minkowski background. As usual, we shall decompose both the electromagnetic perturbation $a_{\mu}$ and the metric perturbation $h_{\mu \nu}$ in Fourier modes and separate them into scalar, vector and tensor contributions (we follow the same procedure as in [11]). The corresponding propagation speeds for the perturbations are:

$$
\begin{aligned}
& c_{s}^{2}=1 \\
& c_{v}^{2}=\frac{1-8 \pi G \sigma(1-2 \sigma) \bar{A}_{0}^{2}}{1-8 \pi G \sigma \bar{A}_{0}^{2}} \simeq 1+16 \pi G \sigma^{2} \bar{A}_{0}^{2} \\
& c_{t}^{2}=\frac{1+8 \pi G \sigma \bar{A}_{0}^{2}}{1-8 \pi G \sigma \bar{A}_{0}^{2}} \simeq 1+16 \pi G \sigma \bar{A}_{0}^{2}
\end{aligned}
$$

where we have expanded for $|\sigma| \ll 1$. Notice that the scalar modes propagate at the speed of light irrespective of the value of the parameter $\sigma$. However, the speed of photons $c_{v}$ would be larger than the "speed of light" $c=1$ which determines the null cones of the Minkowski geometry. This in principle could give rise to inconsistencies with causality in the theory. However, it is known that in scenarios with violations of the strong equivalence principle, as the one considered here, superluminal propagation can be consistent with causality, provided stable causality is ensured [12]. For that purpose, if the new light cone can be written as $\mathcal{G}^{\mu \nu} k_{\mu} k_{\nu}=0$, then there must exist a globally defined function $f$, such that $\nabla_{\mu} f$ must be non-vanishing and timelike everywhere with respect to $\left(\mathcal{G}^{-1}\right)_{\mu \nu}$. In our case, the light cones for vectors can be written as:

$$
\left[\left(1+16 \pi G \kappa \bar{A}^{2}\right) \eta_{\mu \nu}-16 \pi G \kappa \bar{A}_{\mu} \bar{A}_{\nu}\right] k^{\mu} k^{\nu}=0
$$

with $\kappa=\sigma^{2} /\left(1-\sigma A^{2}\right)$, whereas for tensors:

$$
\left[\left(1+8 \pi G \sigma \bar{A}^{2}\right) \eta_{\mu \nu}-16 \pi G \sigma \bar{A}_{\mu} \bar{A}_{\nu}\right] k^{\mu} k^{\nu}=0
$$

Since $\bar{A}_{0} \lesssim M_{P}$ and $|\sigma| \ll 1$, in both cases, the effective metric $\left(\mathcal{G}^{-1}\right)_{\mu \nu}$ is a small perturbation with respect to Minkowski. This implies that we can use the time coordinate $t$ as the globally defined function $f$. Thus, we see that, for small $\sigma$, the theory does not exhibit classical instabilities or causality inconsistencies.

In order to study the presence of quantum instabilities (ghosts), we analyze the positiveness of the energy density of the three types of perturbations considered before. Thus, we define the energy for the modes as $[11,13]$ :

$$
\rho=\left\langle T_{00}^{(2)}-\frac{1}{8 \pi G} G_{00}^{(2)}\right\rangle
$$

where $T_{\mu \nu}^{(2)}$ and $G_{\mu \nu}^{(2)}$ are the energy-momentum tensor of the vector field and the Einstein's tensor calculated up to quadratic terms in the perturbations and $\langle\cdots\rangle$ denotes an average 
over spatial regions. Although the calculation has been performed in the longitudinal gauge, both, mode frequencies and energies, do not depend on the gauge choice.

For scalar modes we find that the energy density vanishes identically if we impose the Lorenz condition, as in ordinary electromagnetism (see [8] for expanding backgrounds). For vector and tensor modes, the energy densities are:

$$
\begin{aligned}
\rho_{v} & =2 k^{2} \frac{1-8 \pi G \sigma\left[2+8 \pi G \sigma(2 \sigma-1) \bar{A}_{0}^{2}\right] \bar{A}_{0}^{2}}{\left(1-8 \pi G \sigma \bar{A}_{0}^{2}\right)^{2}}|\vec{C}|^{2} \\
& \simeq 2 k^{2}\left(1-16 \pi G \sigma^{3} \bar{A}_{0}^{4}\right)|\vec{C}|^{2} \\
\rho_{t} & =k^{2} \frac{1-8 \pi G \sigma\left(2+8 \pi G \sigma \bar{A}_{0}^{2}\right) \bar{A}_{0}^{2}}{1-8 \pi G \sigma \bar{A}_{0}^{2}}\left(\left|C_{\oplus}\right|^{2}+\left|C_{\otimes}\right|^{2}\right) \\
& \simeq k^{2}\left(1-8 \pi G \sigma \bar{A}_{0}^{2}\right)\left(\left|C_{\oplus}\right|^{2}+\left|C_{\otimes}\right|^{2}\right)
\end{aligned}
$$

where $\vec{C}$ is the amplitude of the Fourier mode for the vector modes and $C_{\oplus, \otimes}$ are the amplitudes of the two polarizations of the gravitational waves. From these expressions we see that the theory is also free from quantum instabilities for small $|\sigma|$.

\section{Cosmological evolution}

In the following we shall show that, due to the smallness of the parameter $\sigma$, the cosmological evolution of the homogeneous mode becomes modified in a negligible way by the presence of the coupling to the Einstein tensor. This ensures that the inflationary generation and evolution discussed in [8] is also a good description in the non-minimal case. We shall consider an electromagnetic field of the form $A_{\mu}=\left(A_{0}(t), 0,0, A_{z}(t)\right)$ in a FLRW metric $d s^{2}=d t^{2}-a(t)^{2} d \vec{x}^{2}$. In this case, the equations of motion read:

$$
\begin{aligned}
\ddot{A}_{0}+3 H \dot{A}_{0}+3\left(\dot{H}-2 \sigma_{\lambda} H^{2}\right) A_{0} & =0 \\
\ddot{A}_{z}+H \dot{A}_{z}+\sigma\left(4 \dot{H}+6 H^{2}\right) A_{z} & =0
\end{aligned}
$$

where $\sigma_{\lambda}=\frac{\sigma}{\lambda}$. In a de-Sitter inflationary era with $H=H_{I}$ constant, the growing mode solutions behave for small $\sigma$ as:

$$
A_{0}(t) \propto \exp \left(2 \sigma_{\lambda} H_{I} t\right), \quad A_{z}(t) \propto \exp \left(-6 \sigma H_{I} t\right)
$$

During the radiation and matter dominated epochs in which $H=p / t$ with $p=1 / 2$ and $p=2 / 3$ respectively, the solutions are:

$$
A_{0}(t) \propto t^{1+3 \sigma_{\lambda} / 5}, \quad A_{z}(t) \propto t^{1 / 2+\sigma}
$$

in the radiation era, and

$$
A_{0}(t) \propto t^{1+8 \sigma_{\lambda} / 9}, \quad A_{z}(t) \propto t^{1 / 3}
$$

in the matter era. We see that the the only effect of the non-minimal coupling is a slight modification in the power exponents. Finally, in a universe dominated by the electric potential $A_{0}(t)$, we have a power law expansion of the form $a(t) \propto t^{-\frac{\lambda}{2 \sigma}}$. For small $\sigma$, we have an accelerated expansion which corresponds to a quasi de Sitter phase with slow-roll parameter $\epsilon=-\frac{2 \sigma}{\lambda}$. Notice that, in the limit $\sigma \rightarrow 0$, we also recover the pure de Sitter solution found in the minimal case. 


\section{Effective electromagnetic current: gravitational magnetism}

Let us now consider the possible effects of the new effective electromagnetic current $J_{g}^{\mu}=$ $2 \sigma G^{\mu 0} \bar{A}_{0}$ in (2.2). Using Einstein equations to relate $G^{\mu \nu}$ to the matter content, we obtain:

$$
J_{g}^{\mu}=16 \pi G \sigma T^{\mu 0} \bar{A}_{0}
$$

so that the effective electromagnetic current is essentially determined by the four-momentum density. Moreover, if we assume $T^{\mu \nu}=(\rho+p) u^{\mu} u^{\nu}-p \eta^{\mu \nu}$ at first order, we can see that the energy density of any perfect fluid has an associated electric charge density given, for small velocities, by:

$$
\rho_{g}=J_{g}^{0}=16 \pi G \sigma \rho \bar{A}_{0}
$$

and the three-momentum density generates an electric current density given by

$$
\overrightarrow{J_{g}}=16 \pi G \sigma(\rho+p) \vec{v} \bar{A}_{0}
$$

This theory effectively realizes the old conjecture by Schuster, Einstein and Blackett [14] of gravitational magnetism, i.e. neutral mass currents generating electromagnetic fields. Early attempts to encompass this conjecture in a gravitational theory can be found in [15].

In the case of a particle of mass $m$ at rest, (5.2) introduces a small contribution to the active electric charge (the source of the electromagnetic field), given by $\Delta q=16 \pi G \sigma m \bar{A}_{0} \simeq$ $15 \sigma\left(m / M_{P}\right)$, but does not modify the passive electric charge (that determining the coupling to the electromagnetic field). In fact, this would give different active charges to electrons and protons due to their mass difference and, in addition, would provide the neutron with a nonvanishing active electric charge. However, the effect is very small in both cases $\Delta q \simeq 4 \sigma 10^{-18} e$ where $e=0.303$ is the electron charge in Heaviside-Lorentz units. Present limits on the electron-proton charge asymmetry and neutron charge are both of the order $10^{-21} e$ [16], implying $|\sigma| \lesssim 10^{-3}$ which is less stringent than the PPN limit discussed before. Notice also that photons would acquire a non-vanishing active electric charge. However tight existing limits imposed by deflection of radio pulsar emission by galactic magnetic fields [17] only apply to passive charge which is not modified gravitationally (see also [18]).

On the other hand, for any compact object, even in the case it is neutral, the effective electric current will generate an intrinsic magnetic moment $\vec{m}=\frac{1}{2} \int \vec{r} \times \vec{J}_{g}(\vec{r}) d^{3} \vec{r}$ given by:

$$
\vec{m}=\beta \frac{\sqrt{G}}{2} \vec{L}
$$

with $\vec{L}$ the corresponding angular momentum and $\beta$ a constant parameter whose value is:

$$
\beta=16 \pi \sqrt{G} \sigma \bar{A}_{0}
$$

Notice that relation (5.4) resembles the Schuster-Blackett law, which is an empirical relation between the magnetic moments and the angular momenta found in a wide range of astrophysical objects from planets, to galaxies, including those related to the presence of rotating neutron stars such as GRB or magnetars [19]. Let us mention that the observational evidence on this relation is still not conclusive. From observations, the $\beta$ parameter is found to be in the range 0.001 to 0.1 .

Imposing the PPN limits on the $\sigma$ parameter, we find $\beta \lesssim 10^{-4}$, which is just below the observed range. Thus for a typical spiral galaxy, a direct calculation provides: $B \sim \sigma 10^{-4} \mathrm{G}$, i.e. according to the PPN limits, the field strength could reach $10^{-9} \mathrm{G}$ without amplification. 
However, notice that even in the case in which this generation mechanism took place, the determination of the actual amplitudes of magnetic fields in astrophysical objects would require to take into account the full magnetohydrodynamical evolution. Therefore, we do not expect the gravitationally generated magnetic field to necessarily agree with observations. However this mechanism could help seeding standard amplification mechanisms such as dynamo with appropriate fields correlated to the object angular momentum.

It is also interesting to evaluate the maximum magnetic field that could be generated in a Blackett-like experiment in laboratory [20]. Thus for a rotating neutral sphere of $M=$ $500 \mathrm{~kg}$, radius $R=0.5 \mathrm{~m}$ and rotation frequency $\omega=100 \mathrm{~Hz}$, the field amplitude would be $B \sim \sigma 10^{-10} \mathrm{~T} \lesssim 10^{-15} \mathrm{~T}$, which is just below the fundamental sensitivity limit of SQUID or SERF magnetometers [21].

\section{Discussion}

Notice that in this scenario, it is the non-vanishing Ricci curvature what generates electromagnetic fields in the presence of dark energy. Notice, however, that the only requirement for the main results of the present work is the presence of a cosmological electric potential. This implies that magnetic fields would be associated to the presence of a non-vanishing energy-momentum distribution. In other words, the effect would be absent in vacuum even for curved backgrounds.

Another interesting consequence of the presence of non-minimal couplings in the electromagnetic action (2.3) is the fact that they play the role of an effective mass term for the electromagnetic field during inflation. This naturally provides an infrared cutoff in the calculation of the field dispersion from quantum fluctuations [22].

As shown before, fields of strengths up to $10^{-9} \mathrm{G}$ could be generated on galactic scales in this theory, which could seed a galactic dynamo or even play the role of "primordial" seeds and account for the observed magnetic fields in galaxies and clusters just by adiabatic compression in the collapse of the protogalactic cloud. Therefore, a detailed study of the magnetohydrodynamical evolution in the presence of the gravitationally-induced current will help establishing the importance of dark energy in the origin of cosmic magnetic fields.

\section{Acknowledgments}

We would like to thank Prof. Misao Sasaki for useful comments. This work has been supported by MICINN (Spain) project numbers FIS 2008-01323 and FPA 2008-00592, CAM/ UCM 910309, MEC grant BES-2006-12059 and MICINN Consolider-Ingenio MULTIDARK CSD2009-00064. J.B. also wishes to thank support from the Norwegian Council under the YGGDRASIL project no 195761/V11.

\section{References}

[1] L.M. Widrow, Origin of galactic and extragalactic magnetic fields, Rev. Mod. Phys. 74 (2002) 775 [astro-ph/0207240] [SPIRES];

R.M. Kulsrud and E.G. Zweibel, The origin of astrophysical magnetic fields, Rept. Prog. Phys. 71 (2008) 0046091 [arXiv:0707.2783] [SPIRES]; P.P. Kronberg, Extragalactic magnetic fields, Rept. Prog. Phys. 57 (1994) 325 [SPIRES]. 
[2] A. Neronov and I. Vovk, Evidence for strong extragalactic magnetic fields from Fermi observations of TeV blazars, Science 328 (2010) 73 [arXiv:1006.3504] [SPIRES];

F. Tavecchio et al., The intergalactic magnetic field constrained by Fermi/LAT observations of the TeV blazar 1ES 0229+200, arXiv:1004.1329 [SPIRES];

S. Ando and A. Kusenko, Evidence for gamma-ray halos around active galactic nuclei and the first measurement of intergalactic magnetic fields, Astrophys. J. 722 (2010) L39

[arXiv: 1005.1924] [SPIRES].

[3] E.R. Harrison, Generation of magnetic fields in the radiation era, Mon. Not. Roy. Astron. Soc. 147 (1970) 279; Origin of magnetic fields in the early universe, Phys. Rev. Lett. 30 (1973) 188.

[4] M.S. Turner and L.M. Widrow, Inflation produced, large scale magnetic fields, Phys. Rev. D 37 (1988) 2743 [SPIRES];

K. Bamba and M. Sasaki, Large-scale magnetic fields in the inflationary universe, JCAP 02 (2007) 030 [astro-ph/0611701] [SPIRES];

R. Durrer, L. Hollenstein and R.K. Jain, Can slow roll inflation induce relevant helical magnetic fields?, arXiv:1005.5322 [SPIRES].

[5] J.M. Quashnock, A. Loeb and D.N. Spergel, Magnetic field generation during the cosmological QCD phase transition Astrophys. J. 344 (1989) L49 [SPIRES];

T. Vachaspati, Magnetic fields from cosmological phase transitions,

Phys. Lett. B 265 (1991) 258 [SPIRES].

[6] O. Bertolami and D.F. Mota, Primordial magnetic fields via spontaneous breaking of Lorentz invariance, Phys. Lett. B 455 (1999) 96 [gr-qc/9811087] [SPIRES].

[7] A.L. Maroto, Primordial magnetic fields from metric perturbations, Phys. Rev. D 64 (2001) 083006 [hep-ph/0008288] [SPIRES];

S. Matarrese, S. Mollerach, A. Notari and A. Riotto, Large-scale magnetic fields from density perturbations, Phys. Rev. D 71 (2005) 043502 [astro-ph/0410687] [SPIRES];

K. Ichiki, K. Takahashi, H. Ohno, H. Hanayama and N. Sugiyama, Cosmological magnetic field: a fossil of density perturbations in the early universe, Science. 311 (2006) 827 [astro-ph/0603631] [SPIRES];

K. Ichiki, K. Takahashi, N. Sugiyama, H. Hanayama and H. Ohno, Magnetic field spectrum at cosmological recombination, astro-ph/0701329 [SPIRES].

[8] J.B. Jimenez and A.L. Maroto, Cosmological electromagnetic fields and dark energy, JCAP 03 (2009) 016 [arXiv:0811.0566] [SPIRES]; The electromagnetic dark sector, Phys. Lett. B 686 (2010) 175 [arXiv:0903.4672] [SPIRES]; Dark energy: the absolute electric potential of the universe, Int. J. Mod. Phys. D 18 (2009) 2243 [arXiv:0905.2589] [SPIRES]; J.B. Jimenez, T.S. Koivisto, A.L. Maroto and D.F. Mota, Perturbations in electromagnetic dark energy, JCAP 10 (2009) 029 [arXiv:0907.3648] [SPIRES].

[9] J.B. Jimenez and A.L. Maroto, Cosmological magnetic fields from inflation in extended electromagnetism, arXiv:1010.3960 [SPIRES].

[10] C.M. Will, Theory and experiment in gravitational physics, Cambridge University Press, Cambridge U.K.(1993) pg. 380 [SPIRES].

[11] J.B. Jimenez and A.L. Maroto, Viability of vector-tensor theories of gravity, JCAP 02 (2009) 025 [arXiv: 0811.0784] [SPIRES].

[12] S.W. Hawking and G.F.R. Ellis, The large scale structure of space-time, Cambridge University Press, Cambridge U.K. (1973) [SPIRES]; G.M. Shore, Causality and superluminal light, gr-qc/0302116 [SPIRES].

[13] C. Eling, Energy in the Einstein-aether theory, Phys. Rev. D 73 (2006) 084026 [Erratum ibid. D 80 (2009) 129905] [gr-qc/0507059] [SPIRES].

[14] A. Schuster, A critical examination of the possible causes of terrestrial magnetism, Proc. Lond. Phys. Soc. 24 (1912) 121; 
A. Einstein, Über den Äther, Schw. Naturf. Ges. Verh. 105 Pt. 2 (1924) 85;

S.W. Saunders and H.R. Brown, The philosophy of vacuum, Clarendon, Oxford U.K. (1991) pg. 291 [SPIRES];

P.M.S. Blackett, The magnetic field of massive rotating bodies, Nature 159 (1947) 658.

[15] W. Pauli, On the formulation of the laws of nature with five homogeneous coordinates. Part I: classical theory (in German), Annalen Phys. 18S5 (1933) 305 [SPIRES];

J.G. Bennett et al., Unified field theory in a curvature-free five-dimensional manifold, Proc. $R$. Soc. London A 198 (1949) 39;

A. Papapetrou, A 4-dimensional generalization of Wilson's hypothesis, Philos. Mag. 41 (1950) 399;

G. Luchak, A fundamental theory of the magnetism of massive rotating bodies, Can. J. Phys. 29 (1952) 470;

A.O. Barut and T. Gornitz, On the gyromagnetic ratio in the Kaluza-Klein theories and the Schuster-Blackett law, Found. Phys. 15 (1985) 433 [SPIRES].

[16] Particle Data Group collaboration, C. Amsler et al., Review of particle physics, Phys. Lett. B 667 (2008) 1 [SPIRES].

[17] G. Raffelt, Pulsar bound on the photon electric charge reexamined, Phys. Rev. D 50 (1994) 7729 [hep-ph/9409461] [SPIRES].

[18] C. Caprini, S. Biller and P.G. Ferreira, Constraints on the electrical charge asymmetry of the universe, JCAP 02 (2005) 006 [hep-ph/0310066] [SPIRES];

V.V. Kobychev and S.B. Popov, Constraints on the photon charge based on observations of extragalactic sources, Astron. Lett. 31 (2005) 147 [hep-ph/0411398] [SPIRES].

[19] R. Opher and U.F. Wichoski, Origin of magnetic fields in the universe due to nonminimal gravitational-electromagnetic coupling, Phys. Rev. Lett. 78 (1997) 787 [astro-ph/9701220] [SPIRES];

R. da Silva de Souza and R. Opher, Origin of $10^{15}-10^{16} \mathrm{G}$ magnetic fields in the central engine of gamma ray bursts, JCAP 02 (2010) 022 [arXiv:0910.5258] [SPIRES].

[20] S.P. Sirag, Gravitational magnetism, Nature 278 (1979) 535 [SPIRES].

[21] Y.S. Greenberg, Application of superconducting quantum interference devices to nuclear magnetic resonance, Rev. Mod. Phys. 70 (1998) 175 [Erratum ibid. 72 (2000) 329] [SPIRES].

[22] D.H. Lyth, The curvature perturbation in a box, JCAP 12 (2007) 016 [arXiv:0707.0361] [SPIRES];

K. Enqvist, S. Nurmi, D. Podolsky and G.I. Rigopoulos, On the divergences of inflationary superhorizon perturbations, JCAP 04 (2008) 025 [arXiv:0802.0395] [SPIRES];

Y. Urakawa and T. Tanaka, Influence on observation from IR divergence during inflation. I, Prog. Theor. Phys. 122 (2009) 779 [arXiv:0902.3209] [SPIRES]. 